\title{
Why Hygiea Internationalis?
}

\author{
Jan Sundin
}

$\mathrm{I}$

$s$ there a need for a special journal for the history of public health? And, if so, why in electronic form? There are at least two answers to the last question. First of all, new journals in traditional form are expensive to produce and to distribute. Much more substantial support would have been needed from our sponsors to establish the journal on the market and to test its economic potential. We all know the difficulties in convincing our university libraries to subscribe to new journals, a condition necessary for their survival.

Secondly, the electronic medium offers a number of possibilities not easily available for traditional journals. One is the easy access at no other cost for the consumer than time. A second advantage is the possibility to cut the time - just a few months - between the submission of the manuscript and its publication, which shortens the interval between the first expression of new ideas and findings and the moment they reach the rest of the scholarly world.

We have met colleagues who are doubtful about the value of an electronic medium for the spread of high-quality research in the humanities and social sciences. Besides the arguments already mentioned, we would also like to point out that:

- Linköping University guarantees that each issue of the journal will be available - unchanged - on the Internet for 25 years according to the terms of the contract with Linköping University Press.

- hard copy of the journal is available for institutions and private persons at a reasonable cost.

- authors can order off-prints of their own articles.

Having been the co-ordinators of the International Network for the History of Public Health for some years, we are convinced that there is an audience for a special journal within our particular field. Two years ago the network's second international conference, arranged in conjunction with the Society for the Social History of Medicine in Liverpool, gathered more than 100 participants. So did the 
third conference, held in co-operation with the European Association for the History of Medicine and Health. It was organized admirably in Almuñécar by the University of Granada. Public health issues already appear in many traditional journals, which will certainly also be the case in the future. Hygiea Internationalis sees its role as one of a complement to established journals. It may also serve as a place where information in the field may be found and new material tested.

The study of public health and its history is a truly interdisciplinary field. Not only does it include the study of the health of populations and groups within the population (men, women, children, adults, elderly, social classes, ethnic and occupational groups, etc.) by historical demographers and epidemiologists. It also means the study of ideological, professional and popular perceptions about health and disease and the ways and means to promote health and prevent disease in time and space by collective efforts - sanitary reforms, environmental control, vaccination, eradication of risks at work, campaigns for personal hygiene and healthy lifestyles. Thus, this field attracts the medical profession as well as historians, philosophers, ethnologists, anthropologists, geographers, sociologists, economists and political scientists, to name a few.

Just a couple of decades ago, it seemed as if modern science was on its way to solving most of the major health problems, at least in the industrialized part of the world. Then came the discovery that the almost unlimited potential of medical technology in a society with limited resources created a number of ethical questions, one of them being how to make priorities. HIV and AIDS have taught us that the fight against infectious diseases is not over yet. The outcome of the race between resistant germs and new antibiotics seems uncertain. Tuberculosis is a major problem in less favoured regions of the world. Ethnicity, class, gender and other social variables still manifest themselves in health differences. The re-emergence of infectious diseases among children and the increased mortality rates among adults, especially men, in parts of Eastern Europe show what happens when collective institutions break down in modern societies.

When society changes, the health of its population changes too. New forms of society face new types of health problems. One example is the growing concern about the connection between everyday life (work, family and other arenas) and the potential for human beings - as individuals and collectives - to cope with the situation in a way that preserves health and prevents mental and physical disorders. 'Social stress' has emerged as a term describing mental conditions that destroy health and create diseases of different types among unemployed as well as among relatively prosperous but over-worked groups on a labour market that demands flexibility and high performance under new conditions.

Comparative studies - both qualitative and quantitative - in time and space can provide us with a better understanding of these processes, biological as well as social, cultural, ideological and political. Hygiea Internationalis will be open for con- 
tributions that can help us to exchange research results from different countries and different stages of development. Our first issue contains contributions from two distinguished scholars who have approached public health from different angles, as well as a guided tour through sources for studying public health in London from another.

Dorothy Porter provides a survey of the historiography of public health history from the 'grand narratives of progress' of the 1950s to today's post-modern analyses of discourses and the many approaches to what she defines as 'the history of collective action in relation to the health of populations'. She argues that this history must also be studied in relation to the general systems of welfare provision and the welfare state. She concludes by calling for a dialogue across both methodological, conceptual and chronological boundaries 'from the ancient past even perhaps to speculation upon possible futures'.

Virginia Berridge's article 'History in Public Health' reflects upon the use of public health history by non-historical practitioners from the point of view of a historian who has been working side-by-side with the medical profession, at present at the London School of Hygiene and Tropical Medicine. She describes her role as 'living among the tribe' or as 'a stranger and a friend'. Her article shows how history in public health can shed light upon a number of issues presently debated in public health policy, even if historical experience cannot provide prescriptions for the future.

Andrea Tanner's contribution to the 'Archives section' provides an invaluable guide to available sources for the study of London's public health history. No one authority was responsible for all public health measures, but her article shepherds us through the labyrinth of materials preserved in various archives.

Our next issue will be prepared during the autumn of 1999. In the year 2000 we hope that we will have established routines which will allow us to publish up to four issues per year. It is our sincere hope that we will be able to attract the interest of our colleagues all over the world as readers and as contributors. In addition to complete articles, we will keep the door open for serious comments and replies in order to promote discussion.

We would like to remind you that the journal also has the section 'Research and commentaries', where preliminary research notes/reports of a limited size can be displayed for discussion during six months. Hopefully, this section can become a kind of electronic symposium. There is also a 'workshop' where longer working papers on a single topic may be displayed for comment. In addition, the 'news' section will announce scholarly events and distribute other messages that our INHPH members wish to send out. For information, check the buttons on the homepage.

We kindly ask all of our readers to send - by e-mail or otherwise - remarks and comments about this first issue of the journal so that we can provide you with the best possible forum for scholarly communication. 
We are deeply indebted to Professor Erik Sandewall, Department of Computer and Information Science, who provided us with the software system and who has generously offered his invaluable time and advice. This project could never have been launched without his support. We also thank all members of the editorial board, who have offered their experience during the preparation of the journal. Personally I feel profound gratitude to my co-editor Marie Clark Nelson and our technical editor Peter Berkesand for their enthusiastic, dedicated and skilful work in bringing this first volume to fruition. The following Swedish research foundations have provided financial support for the project: The Bank of Sweden Tercentenary Foundation, Swedish Council for Research in Science and the Humanities, and Swedish Council for Social Research.

Jan Sundin

co-editor, co-ordinator of the INHPH 